\title{
"No hay minas buenas sin pleito". Política, concesiones e instituciones mineras en Jujuy, 1880-1930
}

\author{
María Teresa Bovi" \\ Cecilia A. Fandos**
}

Fecha de recepción: 1 de diciembre de 2017. Fecha de aceptación: 28 de abril de 2018

\begin{abstract}
Resumen
A principios del siglo XX en un pase de revista sobre el despliegue minero de Jujuy se advierte, además de los siempre aludidos frenos provocados por la insuficiencia de capitales disponibles, el retraso tecnológico y las dificultades del transporte y la inexistencia de una institucionalidad que resguarde los derechos y genere obligaciones en los actores mineros. En la temprana década de 1930 se usaba el aforismo "No hay minas buenas sin pleitos" para invocar la seguidillas de disputas que despertaba en la vida local el interés minero -al punto de incidir en la renuncia de un gobernador, tras el asesinato de un minero español. El artículo aborda la problemática de la institucionalidad y la conflictividad minera en la provincia entre 1880 y 1930, para lo cual se determinaron los emprendimientos mineros ensayados, se reconstruyó el origen y aplicación de la normativa respectiva y se estudiaron las prácticas mineras y la injerencia política en el asunto.
\end{abstract}

"There are no good mines without lawsuits". Politics, concessions and mining companies in Jujuy Province 18801930

\footnotetext{
Abstract

At the beginning of the 20th century a review on mining deployment in Jujuy showed -apart from the recession caused by the lack of capital, the technological backwardness and the transportation difficulties- the absence of institutions

" Centro Interdisciplinario de Investigaciones en Tecnologías y Desarrollo Social para el NOA. Unidad Ejecutora. Consejo Nacional de Investigaciones Científicas y Técnicas. Universidad Nacional de Jujuy (CIITED - UE. CONICET/ UNJu) - Unidad de Investigación en Historia Regional - Universidad Nacional de Jujuy (UNIHR/ UNJu). San Salvador de Jujuy, Argentina. E-mail: maritabovi@hotmail.com

* Centro Interdisciplinario de Investigaciones en Tecnologías y Desarrollo Social para el NOA. Unidad Ejecutora. Consejo Nacional de Investigaciones Científicas y Técnicas. Universidad Nacional de Jujuy (CIITED - UE. CONICET/ UNJu) - Unidad de Investigación en Historia Regional - Universidad Nacional de Jujuy (UNIHR/ UNJu). San Salvador de Jujuy, Argentina E-mail: cecifandos@gmail.com
}

\section{Palabras clave}

negocio minero ciclos y ritmo instituciones y conflictos siglo XIX

Key words

mining business cycles and rhythms conflicts and institutions $19^{\text {th }}$ century 
1. Sobre la minería colonial de la jurisdicción de la Gobernación del Tucumán puede consultarse Sica y Ulloa (2006), Gluzman (2007), Gil Montero (2011), Estruch et al. (2011) y Becerra (2012) protecting the rights and promoting obligations among the mining actors. In the early 1930s the aphorism "There are no good mines without lawsuits" was used to invoke the string of disputes the mining interest aroused in local life -to the point that even a governor had to resign after the murder of a Spanish miner. The article discusses the institutional problem and the mining conflict in the province between 1880 and 1930, the approach takes into consideration the mining ventures tested, the reconstruction of regulations - origins and application- and the mining practices and the political interference.

\section{Introducción}

La historiografía de la minería latinoamericana ha revisado el concepto de una crisis minera desatada tras el proceso de la independencia y con pocas posibilidades de una recuperación inmediata. Los estudios abocados a los grandes centros mineros coloniales -México, Perú y Bolivia- han sacado a luz diversos ciclos de auge y de recuperación durante el siglo XIX. Ese resultado, en parte, fue posible por un cambio en la perspectiva teórica y por la multiplicación de estudios empíricos. En efecto, las interpretaciones y las pruebas acerca de la minería decimonónica latinoamericana dependen mucho de dónde coloca el análisis el que mira: si en los patrones económico-productivos y socioculturales fundados en la colonia, la llamada minería tradicional o minería de antiguo régimen, de los metales preciosos y asociada a la pequeña minería de los campesinos excavadores o lavadores; o en los paradigmas de la minería moderna, vinculada al ferrocarril, la explotación de los metales no ferrosos y siderúrgicos -cobre, plomo, zinc, hierro-, la escala empresarial de las compañías internacionales y el sesgo exportador de esta producción (Deustua, 2004). Hoy se comprende mejor, en todo caso, que la minería de América Latina en el siglo XIX sostuvo simultáneamente los dos modelos en un proceso de maduración paulatino del segundo.

La provincia de Jujuy, situada en el extremo noroeste de la Argentina en la zona limítrofe con Bolivia y Chile, fue escenario de una intensiva práctica minera colonial de "antiguo régimen", cuyo polo se ubicó en la región de la Puna. ${ }^{1}$ En la década de 1930 se consolidó en este territorio la mega minería de explotación metalífera, con la puesta en funcionamiento de las plantas de Mina Pirquita -de la firma Pirquitas, Picchetti y Cía- y Mina El Aguilar -a cargo de National Lead Company- (Salizzi, 2014; Gómez Lende, 2016).

El panorama sobre el siglo XIX que se muestra recurrentemente reproduce esta imagen expuesta en 1891:

Fuera de los trabajos de los indígenas, y más tarde de los españoles, trabajos superficiales, que les dieron sin embargo fabulosas cantidades de oro, según las estadísticas, y la misma tradición que queda vida todavía, nadie después ha intentado una explotación racional sujeta a las reglas de la minería moderna (Publicación Oficial, 1891: 30).

Desde este tipo de lecturas la situación de la actividad se califica como ineficiente en términos tecnológicos, insuficiente en los capitales invertidos, con ambientes mineros carentes de recursos imprescindibles como agua y combustible (Publicación Oficial, 1891: 57-62). No obstante, se pondera el esfuerzo público y privado desplegado en el último cuarto del siglo XIX en pos de la modernización, cuyo principal símbolo era el "silbido" de ferrocarril que ya se comenzaba a oír en la región. Pero había un factor de freno tan importante 
como los otros que debía atención urgente y sobre el cuál se emitian fundadas recomendaciones:

[...] una vez en función el ferrocarril el gobierno debe pensar en la organización estricta de oficinas especiales en la que haya un técnico por lo menos que determine las muestras de minerales, que la ley nacional exige [...] aún hay más por hacer: se impone allí obligar a los poseedores de pertenencias a que las amparen y a restringir el número de las que pueden requerir los solicitantes, de acuerdo con el capital de que disponen (Holmberg, [1904] 1988: 19).

La advertencia traza, por un lado, la inexistencia de un cuadro normativo acorde y, por el otro, enumera algunos vicios de la práctica minera. La cuestión institucional que plasma este juicio de época no se resolvió inmediatamente, más allá de las reformas legislativas que se fueron introduciendo. Así, a comienzos de la década de 1930 un informe citaba el aforismo: "No hay minas buenas sin pleitos" para invocar las seguidillas de disputas que despertaba en la vida local el interés minero; al punto de incidir en la renuncia de un gobernador -Arturo Pérez Alisedo-, tras el asesinato de un minero español. ¿En qué radicaba la problemática institucional?, ¿cuáles eran las disputas?, ¿quénes las protagonizaban y porqué? Entendemos que el análisis de los conflictos desatados y su impronta en la normativa minera resulta un aspecto desconocido y puede ser un capítulo interesante en el conocimiento histórico de esta gran "industria" de la etapa transicional del siglo XIX al XX.

Desde esa preocupación principal nos proponemos, en primer término, determinar los diversos emprendimientos mineros entre fines del siglo XIX y la década de 1930; en segundo lugar, reconstruir el tejido institucional de la etapa a través del seguimiento del origen y de la aplicación de la normativa minera, en tercer lugar, establecer las prácticas mineras y las interrelaciones sociales, expresadas a través de los conflictos mineros y, en cuarto lugar, recorrer la injerencia del poder político en la materia siguiendo el tejido de intereses gestado en torno a los pedidos de cateo, explotación y concesiones.

Trabajamos con distinta documentación oficial -informes, leyes, debates parlamentarios, correspondencia, padrones mineros, prensa de la epoca- pero fundamentalmente con un repositorio específico existente en el Archivo Histórico de Jujuy (en adelante, AHJ) catalogado como expedientes mineros, consultados en serie para un período de 50 años (1880-1930) (Anexo 1). El tipo de información que nos proporciona esta fuente no es una estadística de la extracción de minerales o de las empresas del ramo sino de los intereses mineros que se fueron creando. El corpus documental contiene una variedad de asuntos tramitados sobre la materia por particulares: pedidos de cateo, reconocimientos de "despueble" y posterior concesión; descubrimientos, explotación y suspensión de trabajos.

\section{Los emprendimientos mineros en Jujuy. Ritmos y ciclos entre la década de 1880 y 1930}

La actividad minera de la región cobró intensidad desde el último cuarto del siglo XIX y hubo un ciclo expansivo al promediar la década de 1890, seguramente ligado a la proyección y la habilitación del primer tramo del Ferrocarril Central Norte en suelo jujeño. Una de las características centrales fue el progresivo desplazamiento en la importancia de los metales preciosos hacia la exploración y explotación de los metales y substancias industriales. 
Entre 1880 y 1930 los minerales de explotación de Jujuy que más se visualizan en la documentación de la época son el oro, el bórax, el petróleo y el plomo; la dinámica en el tiempo que siguió cada uno de ellos tuvo claros comportamientos, como se refleja en siguiente gráfico (Gráfico 1).

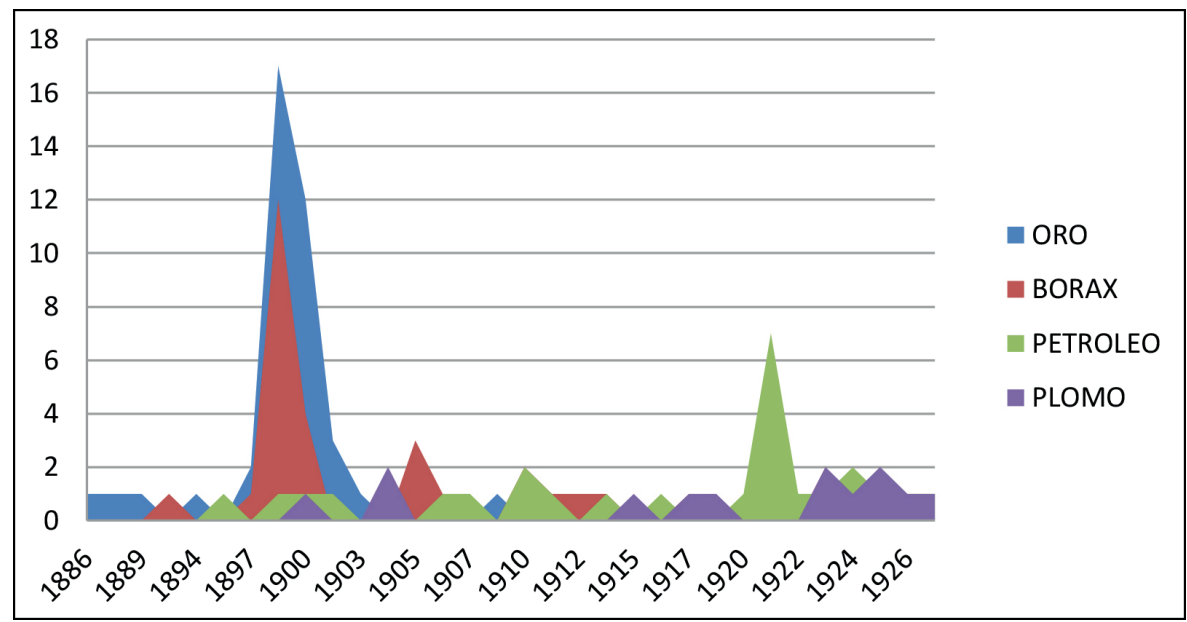

Gráfico 1. Provincia de Jujuy. Ritmos de los minerales solicitados en los expedientes mineros (décadas de 1880 a 1930). Fuente: elaboración propia, en base a fuentes citadas en el Anexo 1.

Se manifiesta el predominio en dos rubros centrales en las décadas de 1890 y 1900: la continuidad del oro y la emergencia del borato. En cambio, el petróleo y en menor medida el plomo, despunta con más notoriedad recién en la década de 1920.

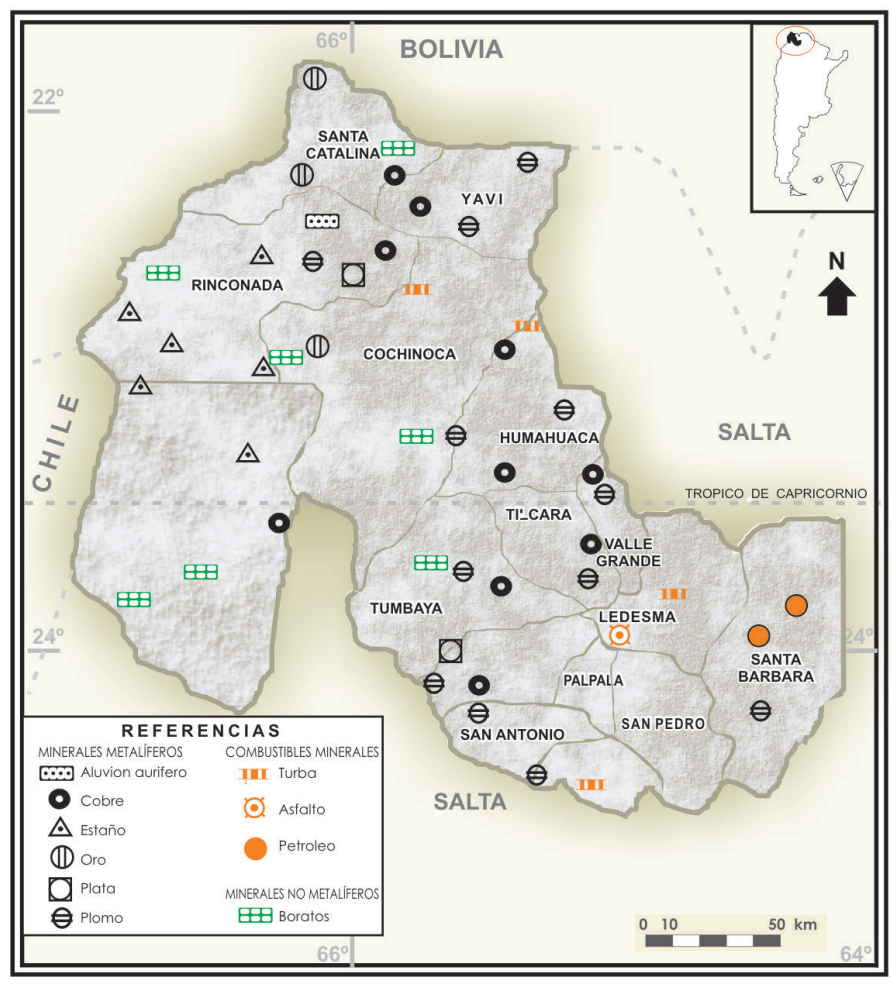

Mapa 1. Provincia de Jujuy. Principales yacimientos mineros hacia la década de 1930. Fuente: elaboración propia, en base a Saravia (1960). 
En la minería el desarrollo de ciertas ramas se vincula muy fuertemente al descubrimiento de nuevos criaderos, pero también a corrientes de intereses en torno a determinadas metales que incentivan las políticas públicas y las inversiones privadas. En este sentido, la explotación del oro en el territorio de Jujuy a finales del siglo XIX fue animada por los varios informes técnicos que se expidieron entonces y que alentaron los flujos de capitales para sacar a flote las indiscutidas riquezas auríferas de Jujuy. Así, las operaciones de inspección minera en la región se intensificaron tanto por la acción privada, encarnada en las figuras de los viajeros, como en las comitivas oficiales gestadas desde el Estado. ${ }^{2}$ A modo ilustrativo traemos a colación el estudio que hiciera el ingeniero de minas Victorio Novarese, en relación a los yacimientos auríferos localizados en la Puna jujeña. En sus observaciones percibe asiduas trazas de antiguas explotaciones, excavaciones, pozos, ruinas de numerosas habitaciones "reunidas a veces en verdaderos pueblos" lo que indica, una vez más, la importancia que tuvo la región puneña en la explotación de la minería aurífera colonial. Además, manifiesta que "Desde hace algún tiempo esos yacimientos llamaron otra vez la atención del público, especialmente desde que los ferrocarriles llegaron a Jujuy y se han practicado nuevos estudios y exploraciones" (Novarese, 1893: 116).

A ese empuje de carácter científico y político se sumó un hecho casual, el descubrimiento de un nuevo criadero de oro, condicionado aún más por el perfil del actor que lo llevó a cabo. El suceso llegó a marcar un verdadero punto de inflexión en la década de 1890 y predispuso un activo entorno publicitario de la minería jujeña. Conviene traer al ruedo una nota periodística publicada en un diario local para divisar la trascendencia del caso y sus proyecciones:

\footnotetext{
Rodeados de inmensa riqueza hemos vivido pobres. Informes de este y de aquel nos decían que teníamos grandes criaderos de oro. Nosotros, incrédulos, no habíamos visto ni tocado nada. Creíamos que eran delirios de cabezas calenturientas. El tiempo ha transcurrido y el descubrimiento de un indio natural de la puna, el indio Canavire, ha ido hasta Europa, su nombre y su mina "La Esperanza" se ha convertido en 10.000 libras esterlinas, y las minas contiguas de "La Fe", "La Piamontesa" y "La Envidiada" -a cuyos dueños se llamaba locos-, esa locura les ha producido la suma de 44.938 pesos moneda legal. Esas cuatro pertenencias negociadas [...], le ha dado 240.000 libras esterlinas. Locuras envidiables por cierto. ${ }^{3}$
}

Como se deduce, la noticia reporta el descubrimiento del criadero de Toconaite, departamento de Yavi, hecho por Miguel Canavire en 1897 -un poblador natural de la Puna, vinculado a una familia de arrendatarios de tierras fiscales pastores de ovejas. En ella también se apunta la operatoria siguiente al acontecimiento donde participaron nuevos inversores, a veces en sociedad con el propio Canavire. Del otorgamiento inicial a éste de la mina "La Esperanza" se sucedieron varias tramitaciones de exploraciones en la zona con disímil suerte, ya que hubo concesiones que fueron aprobadas y otras rechazadas.

El propio Canavire da cuenta de sus posibilidades y limitaciones para absorber la totalidad de las pertenencias que le correspondían por ley -muy probablemente asesorado por quiénes se sentían atraídos en ese particular negocio. El hecho, inducido o no, culminó con su renuncia a los derechos sobre las áreas vacantes a su mina que benefició a terceros. Canavire, además, buscó nuevos socios y al poco tiempo también terminó por trasferir sus propias concesiones. ${ }^{4}$
2. Un detalle de estas visitas y relevamientos puede verse en Alonso (2010).

3. AHJ. Diario El Norte, 29/1/1900, en Expedientes Mineros, Exp. 169 B, 1900.

4. AHJ. Expedientes Mineros, Exp. 66 Z, 1897. 
5. Mercurio. Revista comercial lberoamericana XVI: (208). Barcelona, 1914, "La riqueza mineral argentina" pág. 339.

6. AHJ. Caja de expedientes, Exp. $344 \mathrm{G}, 1913$.

7. AHJ. Expedientes Mineros, Exp. $118 \mathrm{M}, 1911$.

8. Los primeros pedimentos de exploración de ambos sujetos se hicieron en 1891. AHJ. Expedientes Mineros, Exp. 102 B, 1891.
9. Un diario jujeño aludía claramamente a estas condiciones tras reproducir un artículo de la prensa chilena de la epoca. Diario El Norte, 18 de mayo de 1899 , "Sociedad boratera", AHJ Expedientes Mineros, Exp. 105 L, 1899.
Esta fiebre del mineral del oro en Jujuy continuó con fuerza hasta el primer quinquenio de 1900. Una revista catalana trasluce en todos sus términos esa coyuntura, revelando internacionalmente su riqueza. En la nota publicada, Jujuy era presentada como la "provincia del oro" y se aludiá a los descubrimientos hechos en 1902-1903 sobre el río Orosmayo (Rinconada), donde "Una compañía de reciente formación, constituida para explotar esos lavaderos, la 'Orosmayo Gold Dredging Company', tiene puestas en el negocio grandes esperanzas". 5

La fortuna de la afamada compañía fue incierta, aunque trasladó hasta el corazón de la Puna enormes dragas con una fuerte inversión -pues el carril ferroviario aún no había sido completado-, el emprendimiento fue un fracaso por la falta de previsibilidad de la energía necesaria para el funcionamiento de las mismas. Efectivamente las dragas introducidas eran movidas por la energía de carbón, que dependía de las importaciones británicas con los consecuentes incrementos de unos costos inviables. Además por las condiciones ambientales de la Puna tampoco era factible el uso de energías alternativas, como la leña o la hidráulica. La Compañía de Orosmayo construyó acequias para usar la fuerza motriz del río homónimo, pero resultó de un caudal insuficiente. ${ }^{6}$ La empresa quebró y abandonó los trabajos en 1908, y para 1911 sus bienes continuaban embargados. ${ }^{7}$

Pese a la fascinación por el oro, un inédito producto ocupó el primer orden de atracción minera en Jujuy en esta etapa. Se trata del bórax, un cristal blanco que tiene empleos variados en distintas industrias como la cerámica, el vidrio, la química, la farmacéutica y los micronutrientes para fertilizantes.

En la porción nacional del altiplano andino se encuentra una de las tres reservas mundiales de borato -junto a las de EE. UU, Turquía y México. Según Alonso (2010), la era de los boratos comenzó a fines del siglo XIX y se extendió durante 130 años, siendo la actividad más longeva y permanente de la Argentina. En la puna jujeña puede fecharse su incio en 1895, cuando quedó constituida una compañía a nombre de Agustín Borus y Manuel Pinto. ${ }^{8}$ Es indudable que el borato fue el rubro que dio una certera dimensión internacional a la minería de Jujuy a la vuelta del siglo XX, ya que fue el primer producto de exportación de este sector económico articulado con la demanda de bienes y materias primas de la industrialización mundial de la época, mucho antes de implantarse las plantas productoras de plomo, estaño y zinc.

Desde la pionera acción de los actores locales, Borus y Pintos, la riqueza boratera de Jujuy muy pronto capturó la deferencia de un flujo empresarial de envergadura internacional que le dio continuidad a la actividad, aunque bajo particulares condiciones; entre ellas el incremento en la demanda mundial de este recurso y una tendencia a la centralización y monopolización de las empresas borateras. ${ }^{9}$ Esa pauta concentrada del capitalismo mundial coetáneo alcanzó, al poco tiempo, la realidad provincial mediante la actuación de la Compañía Internacional de Bórax (Bovi y Fandos, 2013).

Otros rubros notorios de la actividad minera al correr los primeros años del siglo XX fueron el petróleo y el plomo. Se conoce que el potencial en combustibles de Jujuy y Salta comenzó a apreciarse en la década de 1820, cuando se comprobó la existencia de betún en la conocida "laguna de la Brea" (Yrigoyen, 2007). 
La literatura reconoce otro estadio de propulsión de esta rama minera con el arribo de comisiones de investigación científicas geológicas en la década 1880. Cuenta especialmente la investigación de Brackebusch, originada en una solicitud de apoyo técnico de la empresa Teófilo Sánchez de Bustamante \& Cia., constituida en 1881 (Riccardi, 2017: 178). Brackebusch asintió que "la explotación del petróleo, por medio de perforaciones dirigidas por hombres competentes, darán un resultado magnífico" (Brackebusch, 1883: 50).

Según los expedientes mineros que hemos trabajado, pese la existencia previa de interesados y de firmas exploradoras, fue en la década de 1920 que se notó una clara expansión del petróleo. A escala nacional esa etapa marca un punto de inflexión por la creación de Yacimiento Petrolíferos Fiscales en 1922. Imperó en ese momento un tono especial en la opinión pública que elevó el tema del petróleo a "causa nacional". Por una parte, implicó una primera definición política de los debates desatados en toda la década previa en el Congreso Nacional sobre el grado de incumbencia del Estado en la explotación petrolífera frente al interés privado. En ese contexto se gestó la línea ideológica del "nacionalismo petrolero", coincidente también con el cambio de escenario económico provocado por la Primera Guerra Mundial. Una importante derivación de la coyuntura fue el puntapié para el primer brote de industrialización por sustitución de importaciones, a su vez incidiendo en la demanda de combustibles (Dachevsky, 2014).

En 1924, con la activación desatada con Yacimientos Petrolíferos Fiscales (YPF) el gobierno de Jujuy, a indicación del Ejecutivo Nacional, declaró el territorio zona de reserva para la exploración y explotación del petróleo. ${ }^{10}$ Durante los años de 1926 y 1927, YPF llevó a cabo trabajos de exploración en la localidad de El Quemado realizando la perforación de dos pozos, uno iniciado por parte de Ferrocarriles del Estado. Esas tareas fueron interrumpidas en 1928 y recién en los primeros años de la década de 1930 YPF reinició las tratativas con el gobierno de Jujuy para la firma de un convenio, mediante el cual la Provincia se obligaba a mantener durante diez años la reserva sobre una extensión de 150.000 has., otorgándole exclusividad a YPF en los derechos de exploración y explotación. ${ }^{11}$

En cuanto al plomo, si bien el mismo prevaleció desde mediados de la década de 1920, aparece en el cuadro de intereses de los expedientes mineros ya en el año 1917. En 1923 la Dirección General de Minas, Geología e Hidrología encargó a Roberto Beder un estudio geológico; alli se da cuenta, entre otras cuestiones, de los principales yacimientos de plomo (Beder, 1928). ${ }^{12}$ En su informe se afirma la preponderancia de la Compañía Minera y Metalúrgica Sudamericana S. A. como principal propietaria de los yacimientos. Era una empresa integrada verticalmente, así a la explotación de plomo le sumó la industria metalúrgica estableciendo una fundidora de plomo en Buenos Aires. Otras dos fundidoras había en Jujuy (de Antonio Lavazza) y otra en Mojotoro, Salta, (de O. Levilly y Cia).

\section{Normas, conflictos y prácticas. Un estudio a través de los expedientes mineros}

La documentación analizada revela, entrelíneas, la experiencia minera y expresa el abanico de reglamentación probada para regular un complejo universo social de interrelaciones conflictivas. Los pleitos relatados y las normas invocadas son indicativos de las propias prácticas, tanto aquellas que quebrantaban
10. República Argentina, Archivo Parlamentario del Congreso de la Nación (en adelante RA- APCN) Exp. 8o P, 1933.

11. RA-APCN Exp. 8o PE, 1935.

12. Menciona las minas de Pumahuasi, Sol de Mayo, 9 de julio, Santa Emilia, Cerro Colorado, entre otras diez más. 
13. Imponía la obligatoriedad del trabajo en la mina durante cierto tiempo como requisito para el mantenimiento de las concesiones.
14. AHJ. Expedientes mineros, Exp. 67 B, 1899; Exp. 45 A, 1899.

15. AHJ. Expedientes mineros, Exp. $143 \mathrm{~A}, 1910$. las leyes generando diversas problemáticas como las que fundaron y originaron nuevas normas.

En el umbral del período que estudiamos, la década de 1880, prescribieron dos importantes leyes. A nivel provincial, se buscó un ordenamiento administrativo especial para los trámites mineros creando un Registro Oficial de Minas en 1884. A escala nacional se supera entonces la etapa en la que la actividad carecía de una carta uniforme y esencial para todo el territorio, al establecerse el Código de Minería en 1887.

Pese a los grandes consensos sobre la esencia de esta codificación, un punto de disenso y continuas reformas en el arco temporal de este estudio fue el sistema de pueble. ${ }^{13}$ De hecho, previo al Código había regido en el país -y en Jujuy también se observaba- el sistema de canon establecido en el Estatuto de Hacienda y Crédito de la Confederación Argentina (1868) por lo cual el sostenimiento de los derechos mineros se afirmaba en el pago de un canon o patente. Esa modalidad fue dejada de lado en la primera versión del Código de Minería (1887) pero una reforma nacional de 1917 lo reestableció. Sobre este aspecto en Jujuy se introdujeron modificaciones importantes un poco antes, entre las décadas de 1880 y 1910.

Una de ellas fue en 1913, cuando el gobierno de la provincia sancionó un paquete impositivo que instrumentaba una patente minera, con diferentes escalas según el tipo de trámite -concesión, exploración, transferencias, etc.- y de mineral. Pese a que proclamaba no contrariar el sistema de "amparo por pueble" del Código de Minería reconocía que quedaba desecha de oficio toda solicitud, o gestión judicial o administrativa en materia minera, por incumplimiento del pago de las patentes (Art. 14). Esta Ley $-\mathrm{N}^{\circ} 207,7$ de noviembre de 1912- entró en vigencia en 1915 cuando se logró habilitar un registro de concesión y propiedad minera dependiente de la Contaduría General (Publicación Oficial, 1917). Eminentemente, el criterio fiscal prevaleció como fundamento de la reglamentación y gradualmente el erario público se vio robustecido por este nuevo ingreso, pero hubo otros entretelones que implicaron a la principal empresa minera de ese momento que es importante comentar.

Sin duda, la estructura empresarial más sólida del sector más sólida en la región hasta la década de 1930 fue la compañía explotadora de los boratos, primero de capitales belgas y luego fundida con varios accionistas internacionales. Desde el inicio de sus actividades despertó el celo de los cuadros políticos locales, quienes reaccionaron frente a ciertos comportamientos de la boratera internacional. Uno de los problemas dectados inicialmente radicaba en la conducta empresarial orientada al acaparamiento de pertenencias de grandes dimensiones con difusas demarcaciones, que obstruía a otros a interesados. ${ }^{14}$

Luego, ya en la década de 1910 fueron las deficiencias del sistema de amparo, burlado sistemáticamente por la Compañía Internacional del Bórax, lo que impugnó la dirigencia política. El principal encargado de levantar la voz para esta época y de sostener una lucha firme contra la empresa fue Benjamín Villafañe (Bovi y Fandos, 2013). Para Villafañe la empresa del Bórax repetía un comportamiento especulativo, monopolizador y acaparador: "No hay quien ignore que el propósito de la Compañía [...] no fue otro que el de cerrar una fuente de producción y evitar la competencia del artículo que explota en otros países" ${ }^{15}$ En su mirada, la solución era una prolija aplicación del sistema de pueble, pues estaba convencido de que cuando rigieran verdaderamente "los deberes de amparo como lo determina el Código de Minería se habrá 
restablecido la corriente comercial" ${ }^{16}$ Villafañe denunciaba que el sistema de pueble era fácilmente infringido por la Compañía al sostener un mínimo de presencia para demostrar el laboreo.

La salida institucional de la problemátcia no fue, sin embargo, en la dirección que pretendía Villafañe pues en el contexto de esta batalla contra la boratera es que se puso en vigencia el nuevo gravámen de las patentes mineras, entre 1913 y 1915, mencionadas anteriormente. ${ }^{17}$ La Compañía inmediatamente acogió la normativa y mucho antes de los plazos establecidos declaró todas sus pertenencias adjuntando un plano y los títulos de propiedad de las mismas, operación por la cual se le impuso el valor de 4.960 pesos a cobrar por renta minera pública.

La compañía Internacional de Bórax sostuvo desde ese año, 1914, y hasta 1918 recurrentes pedidos de suspensión de trabajos por la paralización de la actividad mundial a consecuencia de la Primera Guerra, alegando siempre que era la única protección oficial que requería y que ello no afectaba el "interés general", ni del Estado "puesto que este continuará percibiendo la importante cuota con que la Compañía [...] contribuye a la formación del erario por concepto de patente anual". ${ }^{18}$

A estas reformas de orden provincial hay que sumar el hecho de que en 1918 el propio Código de Minería fue enmendado, mediante la Ley Nacional 10.273 que suprimía la institución del pueble e instituía el canon como único medio de perpetuar el derecho de dominio a las pertenencias mineras. La ley provincial 368 de 1918 se vincula a la ley nacional, reactualizando a nivel local las escalas de las patentes que previamente había ordenado la ley 207 -de 1913-, antes comentada. Además introdujo la obligación de inversión de capital mínimo en obras y a plazo, según las distintas categorías -un mínimo de 10.000 pesos en las minas de primera categoría, 3000 pesos en las de segunda, en las concesiones a sociedad 15.000 pesos, etc.- (Publicación Oficial, 1923).

Para empresas como la Compañía Internacional de Bórax la medida parecía ajustarse más a sus intereses, ya que en principio el costo fiscal representado en el canon era más soportable y rentable en determinadas circunstancias que la obligatoriedad del pueble y sus derivaciones en los costos laborales y de obtención de los insumos de producción. ${ }^{19}$ Villafañe catalogó el cambio normativo nacional y provincial de 1918 como un efecto buscado de la empresa boratera, la que en el afán de eliminar la competencia no solo concentró todos lo minerales en explotación sino que "para no trabajarlos, logró del Congreso Argentino una reforma ad-hoc del Código de Minería que sustituyó el deber del pueble por el deber del canon" (Villafañe, 1926: 18).

Más allá de la actuación de la Compañía Internacional de Bórax y la atención dispensada por la dirigencia política jujeña, las prácticas mineras condensaban una secuencia de vicios. Entre ellos: el favoritismo obrado hacia ciertos sujetos en los casos de superposición de intereses mineros; las irregularidades administrativas como el suministro de información y documentación anticipada por parte de los responsables de las oficinas burocráticas mineras a determinados personajes; el clásico "cajoneo" u ocultamiento de expedientes; la implicancia directa de algún funcionario público actuando como juez y parte en los pleitos; ${ }^{20}$ además de la desorganización reinante en el cuidado de archivos y legajos $\mathrm{y}^{21}$ el hábito recurrente de dilatar los trámites de cateo, cautivando por largos períodos la afectación de una pertenencia que podía concernir a muchos.
16. AHJ. Expedientes mineros, Exp. $143 \mathrm{~A}, 1910$.

17. Establecía un régimen de prórroga sin multa y moratoria para el pago de registros y patentes establecidas en la Ley 207. Como el Estado no había podido efectuar los catastros correspondientes, ordenaba la presentación espontánea de los propietarios de minas para declarar sus pertenencias, con presentación de títulos y planos (Publicación Oficial, 1917: 16).

18. AHJ. Expedientes mineros, Exp. 344 C, 1918; Exp. 446 C, 1914 y Exp. $113 \mathrm{C}, 1917$.

19. Para algunos analistas de la época la inactividad de la boratera internacional era un modo de asegurar el control de los precios mundiales en un mercado que controlaba monopólicamente. En este sentido, poner en pleno trabajo las minas de Jujuy podía significar una baja en la rentabilidad por mayor oferta del producto.

20. AHJ. Expedientes Mineros, Exp. 22 C, 1921; Exp. 27 S, 1929; Exp. 215 G, 1899 y Exp. 678 M, 1920.

21. AHJ. Expedientes Mineros, Exp. 115, 1923; Exp. 27 y 28 G, 1921. 
22. AHJ. Expedientes mineros, Exp. 72 R, 1918 y Exp. 143 G, 1921.

23. AHJ. Diario El Día, Jujuy, 12 de diciembre de 1922, "Los trámites mineros en el gobierno actual", Expedientes mineros, Exp. 130 S, 1922.

24. AHJ. Expedientes mineros, Exp. 1 Z, 1921; Exp. 27 y 28 G, 1921.

25. AHJ. Expedientes mineros, Exp. 37 C, 1921.

26. AHJ. Expedientes mineros, Exp. 143 A, 1910. AHJ. Boletín Oficial, 5 de agosto de 1924, p. 206.
Esto último condujo a la introducción de una norma específica en la Ley 368 -de 1918. Se trata del famoso artículo 14, muchas veces referenciado en los expedientes, por el que se estableció la figura de petición minera abandonada -trámites inmovilizados por seis o más meses-, facultando a la autoridad a ordenar su archivo de oficio. Con la puesta en práctica de esta ley notamos que un $40 \%$ de los expedientes tramitados en la década de 1920 pasaron a archivo por caducidad del trámite. Una consecuencia fue el desplazamiento de la corriente de pedimentos desde las zonas libres y no exploradas hacia los que ya tenían antecedentes, pero que administrativamente se consideraron caducas, motivando claros cruces de intereses entre los actores implicados. Los que se vieron afectados por este control arguyeron que las paralizaciones no eran enteramente su responsabilidad sino que se debían a la "morosidad de los tramites en la oficina pública", por no haber un procedimiento marcado que enumere los términos que debe cumplir cada providencia, diligencia y decreto". ${ }^{22}$

La repercusión de la nueva norma de caducidad del trámite minero alcanzó la prensa local, con la clara intención de incidir en la opinión pública. Así, se acusaba al gobierno de turno de operar con absoluta arbitrariedad en la aplicación de la regla, tras detallarse con número y letra los expedientes cuyos titurales eran favorecidos y quiénes eran perjudicados. ${ }^{23}$ Lo cierto es que se desató una ola de conflictos cuando comenzó a aplicarse el artículo $14{ }^{24}$

Otro vicio de especulación muy frecuente fue el reconocido como "juego de nombres" por el cual se alternaba en las solicitudes la composición de los sujetos partícipes, o se daba inicio a los trámites a nombre de otros para -en breve plazo- transferirlos al verdadero interesado, posibilitando grandes concentraciones de propiedad minera. Una vez más, la Compañía Internacional de Bórax fue el agente inicial visualizado en esta práctica y el protagonista directo de la reglamentación jurídica. ${ }^{25}$ La problemática fue bautizada como "el acaparamiento minero" (Publicación Oficial, 1923: 14) y se vehiculizó a través de una campaña discursiva emprendida por Benjamín Villafañe, la interpelación a los poderes nacionales para que intervengan en el asunto y la proposición y ejecución de leyes. ${ }^{26}$

La gran empresa boratera, con su filosofía, entendía que la unidad territorial minera lograda era fruto de compras y adquisiciones que ninguna norma impedía, por lo tanto, absolutamente legal; como lo era el hecho de poder adquir varias casas o varios campos (AA. VV.,1924: 9).

Para dominar las prácticas especulativas por acaparamiento se instituyó el decreto reglamentario del 9 de enero de 1923, a partir del cual se regulaba la cantidad de cateos pertimidos -hasta dos contiguos- y se prohibía la renovación de cateos a personas o grupos que ya los hubiesen tenido. También obligaba a que antes de la publicación y registro de la solicitud se acreditaran los elementos de trabajo y las máquinas. Por el artículo seis, se introducían los pagos previos a la concesión que debían realizarse en concepto de mensura y demarcaciones. La mecánica de este decreto sirvió como un fuerte filtro de los interesados, dejando fuera de escena a los mineros con menor capital. Inicialmente el decreto alcanzaba únicamente los cateos de substancias de primera categoría del distrito petrolero de la provincia -departamentos de Ledesma, San Pedro, Santa Bárbara y El Carmen-, posteriormente se extendió su aplicación a toda la provincia (Publicación Oficial, 1923: 16-20). 
Llegado el año 1924, la introducción de la Ley 525 buscó corregir varios temas de la experiencia minera provincial. Se creaba por primera vez un órgano específico de administración minera: el Departamento de Minas que establecía normas para el control de los expedientes, los registros de exploración y de minas y los libros de entrada.

En esta ley también se retomó parte del decreto para impedir el acaparamiento minero de 1923, limitando la repetición de concesiones a un mismo sujeto en iguales parajes, con la excepción de quiénes hubieran hecho fuertes inversiones, un mínimo de 50.000 pesos en trabajos, edificaciones e instalaciones. Este filtro por capacidad económica de los actores se reforzaba en otros de los artículos que organizaban los gastos y formas de las mensuras exigidas. Además, obligaba a todos los concesionarios a tener domicilio en San Salvador, por sí mismo o por apoderado, como una forma de limitar a los aventureros. ${ }^{27}$

Uno de los principales puntos de conflictividad que se procuró arbitrar con esta ley era evitar, mediante la creación de un órgano independiente, la injerencia que hasta entonces le cabía al Poder Ejecutivo en la materia. Ciertamente no habían sido menores las situaciones de sospechas de arbitrariedad y parcialidad de actores de los grados más altos del poder político, quienes solapaban intereses mineros particulares -como lo demuestra el escandaloso caso del asesinato de Tauler, que trataremos luego, pero esta ley tampoco tuvo mayores éxitos en ese cometido.

Finalmente, la gran batalla de los conflictos mineros en la década de 1920 se libraría en torno a la explotación petrolífera. Se abrió un frente de disputa con intereses encontrados entre poderosos grupos económicos, el poder político provincial y la mayor dirigencia nacional en materia petrolera: YPF. Los antecedentes se sitúan en el año 1924, luego de que la región Norte del país fuera el principal escenario de atención, tras la visita del Director de YPF, Enrique Mosconi. ${ }^{28}$ Por estímulo de este organismo salieron a la luz tres decretos fundamentales: el decreto del 10 de diciembre de 1924 sobre reglamentaciones del trámite minero de hidrocarburos, el decreto de la misma fecha de creación de un área de reserva y el decreto del 1 de enero de 1925 por el que se transfirió a YPF la concesión de 2.000 hectáreas en la localidad de El Quemado.

La primera norma buscaba crear las condiciones de explotación efectiva de la riqueza petrolera y una distribución adecuada, que evite la especulación y el acaparamiento. A decir verdad, su principal destinatario era la empresa Standard Oil. En la visita de Mosconi quedó en evidencia la existencia de 500 tramitaciones mineras petroleras en Salta y Jujuy con fuerte incidencia de esta firma. Para regular la situación, el principal resguardo pasó nuevamente por elevar la capacidad económica de los solicitantes de cateo de petróleo y otras subtancias al exigir la presentación de una boleta de depósito en garantía expedida por la dirección de rentas de la provincia, de 600 pesos para gastos de mensura de la concesión, alcanzado las nuevas tramitaciones y las que ya estuviesen en curso. El incumplimiento del requisito obraba como causa segura y de oficio de caducidad.

Otro punto que seguía similar criterio versa sobre las solicitudes de prórrogas de concesión. Además de los requisitos para comprobar causas justificables y demostrables se exigía una acreditación de la capacidad económica certificable en el material de perforación de pozos disponible, en una caución en efectivo o un titulo de renta nacional a valores fijados por la autoridad minera pero con un techo mínimo de 50.000 pesos.
27. AHJ. Boletín Oficial, 28 de julio de 1924: 380-382.

28. AHJ. Caja de Expedientes, Exp. 48 M, 1926 y Exp. 723 M, 1926. 
29. AHJ. Caja de expedientes, Exp. $46 \mathrm{M}, 1926$.
Por su parte, el otro decreto de diciembre de 1924 suspendía la concesión de nuevos cateos en un área reservada para estudio y exploración exclusiva del gobierno nacional. Así, a comienzo del año 1925 se autorizó a YPF a explotar 2000 hectáreas en la zona de reserva.

Como resultado global de esta política, la puesta en vigencia de la normativa referida significó un verdadero colador de los muchos actores que habían iniciado petición de cateo. El propio Director de Minas informaba que una vez que comenzaron a aplicarse los requerimientos señalados en los decretos de 400 expedientes presentados 385 caducaron, es decir que solo quince se habían colocado dentro de las exigencias establecidas. A la vez, no se había otorgado nuevas concesiones..$^{29}$ Analicemos el perfil de los únicos 15 actores que lograron alcanzar los nuevos requisitos.

\begin{tabular}{|c|c|c|c|}
\hline $\begin{array}{c}\text { Inicio del } \\
\text { expediente }\end{array}$ & Titulares & Hectáreas & Estado del expediente \\
\hline 1924 & Walterio y Esteban Leach & 1450 & Rectificación \\
\hline 1924 & Esteban Leach y GJ Kelly & 1000 & Demarcación \\
\hline 1924 & Walterio Leach y A Alexander & 500 & Demarcación \\
\hline 1924 & J. Riobo y A Gutiérrez & 1000 & Demarcación \\
\hline 1924 & G J Kelly y A Alexander & 2000 & Rectificación \\
\hline 1924 & R Campos y J. Riobo & 2000 & Rectificación \\
\hline 1924 & R Campos & 500 & Demarcación \\
\hline 1924 & R Campos y J. Riobo & 1500 & Rectificación \\
\hline 1924 & Walterio y Normando Leach & 2000 & Rectificación \\
\hline 1924 & Esteban Leach y GJ Kelly & 2000 & Demarcación \\
\hline 1924 & Walterio Leach y A Alexander & 1925 & Rectificación \\
\hline 1921 & Walterio y Esteban Leach & 2000 & Rectificación \\
\hline 1924 & Salvador Martínez & 500 & Demarcación \\
\hline 1923 & FFCC del Estado & 1757 & Paralizado \\
\hline 1918 & FFCC del Estado & $\mid----$ & Paralizado \\
\hline
\end{tabular}

Cuadro 1. Provincia de Jujuy, Expedientes de cateo de petróleo en tramitación, 1926. Fuente: elaboración propia (Fuente: AHJ. Caja de Expedientes, Exp. 46 M, 1926).

Lo explicitado en el cuadro habla por sí solo. Salvo los expedientes que atañen a los Ferrocarriles del Estado, los otros nuclean distintas asociaciones interconectadas a dos emporios económicos presentes en la región: los propietarios del Ingenio Azucarero La Esperanza y la Standard Oil, en un radio de influencia de 20.000 hectáreas concedidas. Hay que destacar que el grueso de estas tramitaciones se acrecentaron entre los meses de agosto y septiembre del año en que se sancionaron los decretos regularorios de la explotación petrolífera, justo al tiempo que Mosconi se apersonaba en la provincia en pos de la 
campaña defensora de los "intereses nacionales" en el petróleo. No creemos que ello pueda ser producto de una simple coincidencia sino, tal vez, del peso de la influencia de estos actores, principalmente en el manejo de información.

Por su parte, lógicamente, estos actores pudieron hacer frente con bastante soltura a las erogaciones que comenzaron a requerirse. Sin mbargo, el problema radicaba en que había que mensurar el área de reserva de YPF en la concesión otorgada en El Quemado, que en algunos casos se superponía con la de los hermanos Leach y sus accionistas de la Standad Oil. En los informes del director de minas de la provincia, la principal razón que obró en la demora de esta demarcación fue que algunas concesiones de los Leach y la petrolera internacional tenían tramitadas rectificaciones en su ubicación.

Para la administración de YPF, en cambio, los pedidos de rectificación del lugar eran solo una estrategia dilatoria para ganar tiempo e impedir la caducidad por parte de los empresarios implicados. YPF presionaba a traves del Ministerio del Interior de la Nación, incriminando en una serie de informalidades y arbitrariedades a las autoridades mineras de Jujuy y Salta con el fin de interpelar a las gobernaciones de ambas provincias al cumplimiento de los decretos. Los cargos que se les imputaba eran la inacción, la venia a la Standad Oil por acaparamiento de toda la zona petrolífera, la inobservancia de las leyes. ${ }^{30}$

Las controversias se desataron en diversos planos. Por un lado, el pedido de rectificación de ubicación por parte de los Leach desató un conflicto a nivel local por la diferencia de interpretación entre el fiscal general y el director de minas. Hubo varios fallos con otras tantas apelaciones para quedar, al final, facultada la rectificación de lugar solicitada solo en la competencia del Tribunal Superior de Justicia. ${ }^{31}$ Por otro lado, el asunto se manifestó también en torno a la jurisdiccionalidad y competencia de YPF para interpelar, juzgar e incidir en materias que concernían a la Dirección de Minas -y principalmente al gobierno provincial.

Al final, la dirigencia provincial se alineó a favor de los poderosos industriales azucareros, aplaudiendo el emprendimiento conjunto de la sociedad que los Leach habían entablado con la firma extrajera de petróleo. Sobre todo se expresó la posición a favor de Leach y compañía, apartándose de la prédica de YPF:

Debo hacer notar que los señores Leach's hermanos son industriales intensamente vinculados al progreso de la provincia, cuyos establecimientos representan un valor mayor a los 60 millones de pesos, que pagan a la nación en conceptos de impuestos internos alrededor de cuatro millones de pesos al año y cerca de dos millones a la provincia. En sociedad con la Standard Oil o sin ella, están en condiciones de hacer frente a los gastos de explotación. ${ }^{32}$

\section{El gran escándalo: el asesinato del minero Tauler}

A comienzos de la década de 1930 se descubrió placeres de estaños en el departamento de Rinconada y en 1935 comenzó la explotación tras el hallazgo realizado por parte de la Sociedad Minera Pirquitas "Picchetti y Cia" (Rosas y Ávila, 2013). Esta Sociedad quedó registrada como una empresa de exploración y explotación minera el 17 de enero de $1935 .{ }^{33}$. Si bien para esta fecha le fueron otorgadas a la compañía minera las concesiones de estaño, años antes se habían presentado a la Dirección de Minas una serie de pedimentos de cateos. Entre ellos el de Rafael Tauler Andreu, español oriundo de Felanix.
30. AHJ. Caja de expedientes, Exp. $46 \mathrm{M}, 1926$.

31. AHJ. Expedientes mineros, Exp. 27 L, 1921 y Exp. 46 M, 1926.

32. AHJ. Caja de expedientes, Exp. $46 \mathrm{M}, 1926$.

33. Poder Judicial de la Provincia de Jujuy, Juzgado del Comercio (PJPJJC). Registro Público de la Provincia de Jujuy. Libro II, f. 357. El primer Directorio de la Compañía quedo constituido por Walterio Leach como presidente; Gordon Leach como vicepresidente; Gerardo A. Capes como secretario; Alberto Picchetti, Andres Galinzki y Juan Tagliabue como directores; Gerardo A. Capes y Philip W. Brundell como directores suplentes; Enrique A. Dionisi como síndico y Alfredo Reader como síndico suplente. Su representación legal fue conferida a los doctores Eduardo Uriondo Tochón y Mario Busignani y al procurador Martin Zenarruza. (Archivo de Tribunales de la provincia de Jujuy -en adelante, ATJ- Homicidio de Rafael Tauler, 1935, Orden: 799 y ATJ, Homicidio de Rafael Tauler, año 1935, Orden:79). 
El 7 de mayo de 1931 Tauler solicitó permiso para efectuar exploraciones y cateos en busca de minerales de primera categoría, con excepción de petróleo y demás hidrocarburos fluidos, en el cerro Cabalonga (Rinconada) sobre una superficie de 500 has. (Villafañe, 1938 y 1940).

Sin embargo, cuando el expediente pasó al Departamento de Obras Públicas se trabó, precisamente porque se cuestionaron los datos de su localización. Tauler procuró dar solución brindando la nueva información requerida; mientras este trámite corría, según la administración oficial, hubo otro pedido de cateo de Andrés Galisnki -para el grupo de la Compañía de Mina Pirquita- a quién se le cedió por estar libre de superposición. En consecuencia, quedó sin efecto el pedimento de Tauler con el argumento de que se superponía con el de Galisnki y porque había sobrepasado el tiempo de tramitación según el Código de Minería, bajo la figura de incurrencia e inactividad. La situación generó una nueva tramitación de Tauler, nombrando como representantes legales a Pedro Buitrago y Rogelio Alvarado (Buitrago, 1939).

En 1932 a pedido de Julio Figueroa -propietario de los yacimientos minerales colindante a mina Piquitas-, Rafael Tauler fue contratado para cuidar sus pertenencias mineras en Orosmayo. En ese derrotero personal, dos años después, en un círculo de confianza Tauler manifestó temor por su vida, producto de amenazas recibidas, según él, encabezadas por el gobernador de la provincia Pérez Alisedo y Alberto Picchetti; argumentando que: "por ser yo el que di noticias a don Julio Figueroa de la rica mina [...] de Orosmayo, y [...] por obstaculizar los turbios manejos de los señores arriba mencionado" (Villafañe, 1940: 85).

Definitivamente, el supuesto presagio se cumplió y Rafael Tauler fue asesinado por efectivos de la policía durante un traslado, tras haber estado más de doce horas detenido en la comisaría del Salitre perteneciente a la Compañía minera de Pirquitas, por sospechas de que fuese a cometer un atraco. El testimonio y justificativo de aquellos que lo ultimaron, Armando Armando, Antonio Di Santo y Dionisio Zerpa, fue que el propio Tauler extrajo un revolver que llevaba oculto en la pierna y abrió fuego contra sus captores, quienes se vieron obligados a ultimar al atacante en defensa propia.

La muerte del minero Rafael Tauler trajo una peculiar repercusión pues el Gobernador de la Provincia fue acusado de ser el autor intelectual del crimen. Benjamín Villafañe, desde su rol de senador nacional por Jujuy, activó las demandas contra el mandatario e instaló su propia versión de los hechos. En efecto, para él lo declarado por los victimarios era un relato absurdo e incoherente, así como también la detención previa de Tauler y sus peones. Sostuvo que Tauler fue asesinado por los policías bajo encargo del Gobernador Pérez Alisedo, puesto que "Tauler era, sin duda, para los que ambicionaban la posesión de "Pirquitas", un vecino molesto", como cómplice y testigo de sus manejos y atropellos (Villafañe, 1938: 11).

Villafañe enmarca la muerte del minero como un episodio acorde a los continuos incumplimiento a las leyes, incluyendo al Gobernador y sus "cómplices títeres", entre los cuales menciona a la policía, los fiscales del crimen, la Dirección de Minas, entre otros. Por otra parte, acusó la pasividad y falta de interés del Gobernador en colaborar con la justicia, para Villafañe se trataba de un homicidio que tenía por móvil un interés personal, fundado en la disputa de la pertenencia minera (Villafañe, 1938: 18). 
Más allá de su relato, sin duda, en el cruce de acusaciones también incidió una conflictiva trama política. Arturo Pérez Alisedo ejerció la gobernación de Jujuy en 1934, siendo candidato del Partido Popular. Este partido era el fruto de la fusión del conservadurismo local con una fracción del radicalismo antipersonalista que se había constituido tras el derrocamiento del gobernador yrigoyenista Miguel Tanco, a fines de 1930 (Kindgard, 2007: 43).

Por su parte Benjamín Villafañe había iniciado su militancia política en 1906 en las filas del Partido Provincial, incorporándose a partir de 1912 al radicalismo jujeño. Disidente y opositor del radicalismo yrigoyenista fundó en la década de 1920 el Partido Radical Principista (Fleitas, 1997: 12-13). Fue gobernador de Jujuy entre 1924 y 1927 y tras el golpe militar de 1930 se incorporó al Partido Popular, adherido a la Federación Democrática Nacional (Fleitas, 1997: 15). Tres años antes de la muerte de Tauler, Villafañe fue elegido senador nacional (1932-1941), habiéndose desempeñado durante la gestión de Pérez Alisedo como convencional constituyente para la reforma de la Constitución Provincial de 1935.

Los simpatizantes del Partido Popular que respaldaban a Pérez Alisedo aseveraron que la injuria llevada a cabo por Villafañe en contra del mandatario tuvo por móvil "provocar un escándalo para llamar la atención sobre su persona y procurar así la prolongación de su mandato como senador nacional" (Villafañe, 1938: 12). La respuesta de Pérez Alisedo fue demandar a Villafañe por el delito de calumnia, afirmando que las acusaciones en su contra se basaban solo en afirmaciones verbales o en dichos de terceros pero sin pruebas verdaderas. A la par que iniciaba la demanda presentó su renuncia al cargo de Gobernador de la Provincia el 29 de enero de 1936, manifestando que era objeto de una campaña de difamación (Pérez Alisedo, 1937).

Los letrados defensores de Pérez Alisedo insistieron en que Villafañe no pudo probar la supuesta disputa entre el gobernador y Tauler, ni que este último tuvo mejor derecho a la pertenencia a la mina como que tampoco fue descubridor del estaño existente en la mina Pirquitas; por el contrario, insistieron en que Tauler fue un simple empleado que no poseía una posición social tan elevada e importante como la que le atribuyó Villafañe (Ramos y Bonorino, 1938; Alcoba y Uriondo Tochón, 1936 y 1937).

En definitiva, pese a que el asunto impactó fuertemente en la gobernabilidad provinciana, no se pudo probar mayor implicancia de Pérez Alisedo y la afanada Mina Pirquita quedó en propiedad de poderosos actores locales.

\section{Conclusiones}

Sobre una etapa de la mineria jujeña menos conocida, desestimada desde las lecturas ancladas en relatos ponderativos de la "minería moderna", hemos procurado en este estudio arrojar ciertas luces acerca de las características de los negocios mineros a través de los diversos emprendimientos, las políticas mineras diseñadas, así como de las instituciones creadas y las interrelaciones sociales. Con las pistas develadas nos ha sido factible reconocer el conflictivo tejido social de un universo minero muy activo y muy presente en la sociedad, la economía y la política jujeña, entre mediados del siglo XIX y los años iniciales de la década de 1930. 
Aunque quizá el tipo de información básica analizada deja fuera de la mira aquel mundo minero de antiguo régimen, al centrase en los canales formales de la actividad queda claro una paulatina transformación del tipo de minería dominante, desde la de los metales preciosos a la expansión de los boratos, plomo y combustibles. A la par de ese proceso, se fueron configurando estructuras empresariales nóveles: La Compañía Internacional de Borax, la sociedad de los Leach con la Standard Oil, la Empresa Mina Pirquita.

Con el ejercicio de su poder, ciertos rasgos de la minería local persitieron a través de diversas prácticas -como el acaparamiento, el favoritismo, el clientelismo- en un marco administrativo, por lo general, ineficiente.

La experiencia boratera de la década de 1910 -con una fuerte concentración de minas y llevando al mínimo necesario la explotación para sostener el control de los precios en el mercado mundial-, sirvió para que luego los cuadros de la dirigencia local vaticinaran iguales riesgos en los intereses petrolíferos de la década de 1920. Finalmente los problemas institucionales de fondo se tradujeron, en la dramática Década Infame, ya en sucesos de caráceter extraordinarios como el crimen de Tauler.

La documentación analizada revela, entrelíneas, la experiencia minera y expresa el abanico de la reglamentación probada para regular un complejo universo social de interrelaciones conflictivas. Nos interesa puntualizar que lejos de imperar la obsolescencia y rigidez jurídica, el proceso minero de la etapa se sostuvo en base a una institucionalidad dinámica y cambiante, particularmente activa en las administraciones de los gobiernos radicales de la provincia (1918-1930).

Desde la década de 1880 la evolución de la legislación minera giró de la observancia del mecanismo de amparo minero, por el sistema de pueble, a otro medido por la capacidad económica de los actores, cerrando de este modo el círculo de participación en el negocio minero según la riqueza y capital de los sujetos. En la nueva dinámica,como sucedió en gran parte de América Latina, también ganaron los principales emporios del borato, el petróleo y, luego, el estaño.

El "drama del Far West de la puna jujeña", con niveles extremos en la década de 1930 -por la muerte de una persona y la renuncia del jefe de un gobiernoy visibilizado por Benjamín Villafañe a través de sus denuncias, fue parte de los entretelones de la vida política jujeña; estuvo atravesado por la dinámica de una institucionalidad y una práctica minera que se fue fundando en el trayecto de la centuria iniciada en 1900. Efectivamente, en el resonado caso de la década de 1930, desde la mirada nacional se señaló la serie de deficiencias que presentaba la administración y la gestión minera de la jurisdicción. En el informe de Luis Drago se enumeraron, primero, deficiencias en la ubicación geográfica de los pedimentos, competencia que se delegaba a una dependencia "extraña" al Departamento de Minas, como fue la Oficina de Obras Públicas; segundo, carencia de personal legal y técnico idóneo para las tareas de inspección minera; tercero, adulteración de la normativa sobre duración de los cateo -mediante la burla habitual de renuncia y solicitud simultánea por interpósita persona-; cuarto, improcedencia en el otorgamiento de concesiones sin que se haya practicado antes mensura y; quinto, incumplimiento de las leyes sobre obligatoriedad de publicación anual o semestral de padrones de minas (Pérez Alisedo, 1937: 23-41). 
Desde una mirada más especializada en la topografía el informe de Orlando Carnacini habla: en primer lugar, de la imprecisión y vaguedad geográfica de los pedimentos de cateo por carencia de planos de la región, agudizado con la intesificación de la actividad minera, provocando claros casos de superposición. En segundo lugar, se refiere a la realización de pericias incompletas en los procedimientos de mensura, deslinde y amojonamiento; en tercer témino, a la parcialidad de criterio del departamento de minas para el otorgamiento de concesiones; en cuarto lugar, a la cesión de concesiones de superficies por encima de lo fijado por ley en perjuicio de otros mineros, y finalmente, observa las arbitrariedades y abusos de la firmas mineras en el territorio donde se encuentran establecidas. Alude en este caso en forma específica a las tranqueras instaladas en los caminos de acceso a Mina Pirquita, a cargo de servicios de vigilancia privada que impiden el tránsito libre y exigen autorización de la empresa (Pérez Alisedo, 1937: 45-68).

Aunque el marco jurídico-institucional y el espíritu general dominante de la legislación en materia minera fue el de garantizar del modo más eficaz los derechos adquiridos y propender al fin primordial que inspiran todas sus disposiciones: que las minas se trabajen; las realidades descritas ratifican, de una u otra manera, las expectativas, desconfianzas, tensiones y conflictos suscitados en el derrotero que la actividad minera debió afrontar en el periodo analizado.

\section{Anexo: Expedientes mineros del AHJ analizados, agrupados por décadas}

\begin{tabular}{|c|c|}
\hline Décadas & AHJ, Expedientes Mineros \\
\hline 1880 & Expedientes: 7 N 1886, 69 T 1887, 16 O 1888, 4 T 1888, 18 J 1888, 19 J 1888, 2 C 1899, 43 S 1889. \\
\hline 1890 & $\begin{array}{l}\text { Expedientes: } 102 \text { N 1891, } 36 \text { G 1891, } 31 \text { G 1891, } 6 \text { S 1891, 45 S 1892, } 31 \text { L 1894, 150 O 1895, } 106 \text { T 1896, 66, Z 1897, } 116 \text { B 1897, } \\
\text { 117 B 1897, } 121 \text { B 1897, } 36 \text { F 1897, } \\
\text { 62 Z 1897, } 32 \text { B 1898, } 94 \text { M 1898, 4313 B 1899, 46 B 1899, 41 B 1899, 48 B 1899, 55 B 1899, } 56 \text { B 1899, 59 B 1899, 64 B 1899, } 65 \\
\text { B 1899, 69 B 1899, 27 B 1899, 77 B 1899, 78 B 1899, } 94 \text { B 1899, 102 B 1899, 107 B 1899, 120 B 1899, 216 G 1899, 205 L 1899, } \\
\text { 355 C 1899, } 281 \text { C 1899, 365 C 1899, 376 C 1899, 378 C 1899, 380 C 1899, 336 C 1899, 40 S 1899, 45 A 1899, 303 CH 1899, } 304 \\
\text { CH 1899, } 28 \text { Z 1899, 98 T 1899, 65 B 1899, 67 B 1899, 164 B 1899. }\end{array}$ \\
\hline 1900 & 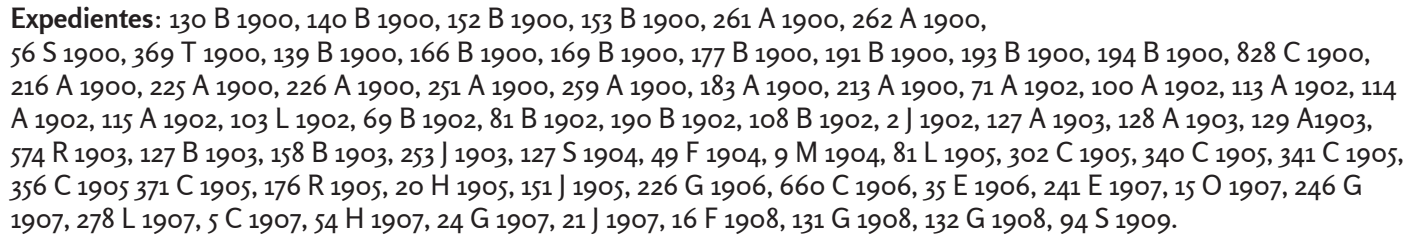 \\
\hline 1910 & $\begin{array}{l}\text { Expedientes: 141 C 1910, } 5 \text { R 1910, 143 A 1910, } 2 \text { A 1911, } 23 \text { A 1911, } 41 \text { A 1911, } 34 \text { S 1911, } 45 \text { C 1911, } 118 \text { M 1911, } 187 \text { M 1911, } 5 \\
\text { O 1912, } 588 \text { C 1912, } 295 \text { M 1912, } 4 \text { E 1913, } 44 \text { L 1914, 446C 1912, } 92 \text { S 1915, 25 F 1915, 427 M 1915, 259 T 1915, 285 A 1916, 230 L } \\
\text { 1916, 154 A 1917, 233 A 1919, } 121 \text { C 1917, } 113 \text { C 1917, } 148 \text { C 1917, } 32 \text { C 1917, S/ L 1917, 89 S 1917, 51 M 1917, 64 M 1917, 19 Z 1917, } \\
\text { 7 T 1917, } 235 \text { G 1917, 1 J 1917, } 12 \text { O 1918, } 19 \text { O 1918, } 344 \text { C 1918, } 139 \text { L 1918, } 246 \text { T 1918, } 30 \text { D 1918, } 52 \text { C 1818, 255 A 1919, 769 C } \\
\text { 1919, 213 R 1919, 69 S 1919, } 24 \text { E 1919. }\end{array}$ \\
\hline 1920 & 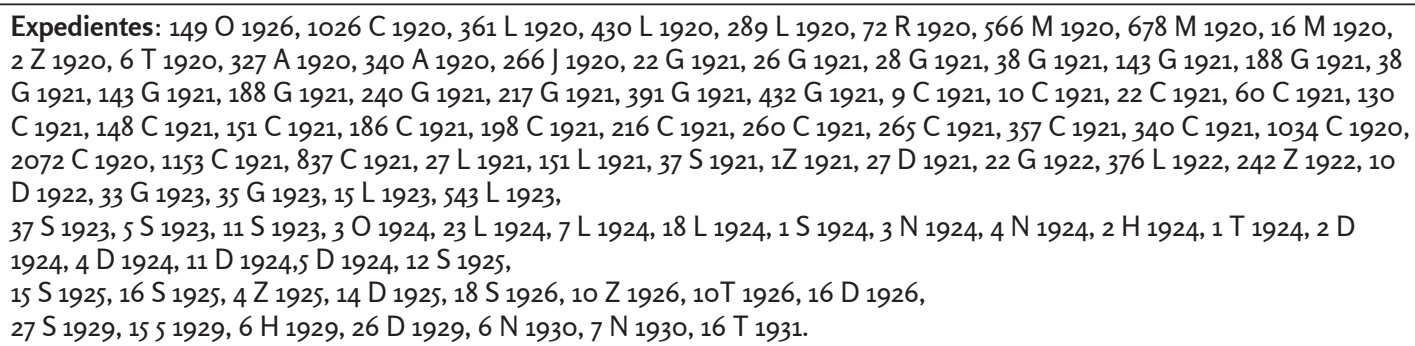 \\
\hline
\end{tabular}




\section{Fuentes consultadas}

》 Archivo Histórico de Jujuy (AHJ)

AHJ. Expedientes Mineros (Ver Anexo)

AHJ. Caja de expedientes. Exp. 344 G, 1913.

AHJ. Caja de expedientes. Exps. 46 M, 48 M y 723 M, 1926.

AHJ. Boletín Oficial, 5 de agosto de 1924: 206.

AHJ. Boletín Oficial, 28 de julio de 1924: 380-382.

»República Argentina - Archivo Parlamentario del Congreso de la Nación (RA-APCN)

RA- APCN Exp. 8O P, 1933.

RA-APCN Exp. 8o PE, 1935

" Poder Judicial de la Provincia de Jujuy, Juzgado del Comercio (PJPJ-JC). Registro Público de la Provincia de Jujuy. Libro II, f. 357.

" Mercurio. Revista comercial Iberoamericana XVI (208). Barcelona, 1914, “La riqueza mineral argentina": 339. 


\section{Q Bibliografía}

" AA.VV. (1924). La Compañía Internacional de Bórax ante el Exmo. Sr. Gobernador de la Provincia, Buenos Aires, Juan Kidd y Cía.

» Alcoba, E. M. y E. Uriondo Tochón (1936). El querellante particular en los delitos de acción pública. Improcedencia de su intervención. Proceso por homicidio a Rafael Tauler. Jujuy, Imprenta B. Buttazzoni.

"Alcoba, E. M. y E. Uriondo Tochón (1937). Proceso por homicidio a Rafael Tauler. Alegatos y sentencia definitiva. Buenos Aires, Imprenta La Británica.

" Alonso, R. (2010). Historia de la minería de Salta y Jujuy, Siglos XV-XX. Salta, Mundo Editorial.

" Becerra, M. F. (2012). Cruces entre la arqueología y la historia: las prácticas minero metalúrgicas en la Puna de Jujuy a través del complejo de fundiciones 1 (Rinconada, Jujuy, Argentina). Población y Sociedad, 19 (1): 5-39.

" Beder, R. (1928). Los yacimientos de mineral de plomo en el departamento de Yavi de la provincia de Jujuy. Buenos Aires, Talleres Gráficos del Ministerio de Agricultura de la Nación.

» Bovi, M. T. y C. Fandos (2013). Riqueza muerta por un trust extranjero. Desarrollo y problemática de la minería boratera en Jujuy (1880-1930). H-industri@ Revista de historia de la industria, los servicios y las empresas en América Latina 13: 1-30.

"Brackebusch, L. (1883). Estudios sobre la Formación Petrolífera de Jujuy. Anales de la Sociedad Científica Argentina 15: 19-58.

" Buitrago, P. (1939). El Gobernador Buitrago contesta las acusaciones formuladas por el Senador Villafañe con motivo de la tramitación de un expediente minero. Jujuy: Imprenta B. Buttazzoni.

"Dachevsky, F. G. (2014). Estado y propiedad del petróleo en Argentina. Antecedentes al surgimiento de Yacimientos Petrolíferos Fiscales (1907- 1922). Revista de Historia Industrial 5: 39-73.

》Deustua, J. (2004). La minería en las Américas en el siglo XIX: el desarrollo de economías sociales. Historias. Revista del Instituto Nacional de Antropología e Historia 58: 83-95.

»Estruch, D., L. Rodríguez y M. F. Becerra (2011). Jurisdicciones mineras en tensión. El impacto de la minería en la Puna jujeña y el valle de Yocavil durante el periodo colonial (Siglos XVII y XVIII). Revista Histórica 35 (2): 69-100.

»Fleitas, M. S. (1997). El pensamiento político y económico de Benjamín Villafañe. Jujuy, UNJu-UNIHR.

» Gil Montero, R. (2011). "El geólogo alemán Ludwig Brackebusch y el "mito" de los mineros jesuitas a fines del siglo XIX en el Noroeste argentino" en Chicote, G y B. Göebel (comps.), Ideas viajeras y sus objetos: El intercambio científico entre Alemania y América austral: 209-219. Madrid y Frankfurt; Vervuert.

» Gluzman, G. (2007). Minería y Metalurgia en la gobernación del Tucumán (siglo XVI y XVII). Memoria Americana. Cuadernos de Etnohistoria 15 (1): 157-184.

"Gómez Lende, S. (2016). Minería metalífera en la provincia de Jujuy, Argentina. Una historia de acumulaión por desposeción (1933-2006). Revista de Estudios Sociales Contemporáneos 15:34-59. 
» Holmberg, E. A. ([1904] 1988). Investigación agrícola en la provincia de Jujuy. Jujuy, Universidad Nacional de Jujuy.

» Kindgard, A. (2007). La Restauración Conservadora en Jujuy. Formas del juego político en tiempos de crisis económica y social, 1930-1943. Travesía 9: 41-66.

» Novarese, V. (1893). Los Yacimientos auríferos en la Puna de Jujuy. Apuntes geológicos. Anales de la Sociedad Científica Argentina 35: 89-116.

»Pérez Alisedo, A. (1937). Resultado de la investigación practicada en la Dirección de Minas de la Provincia de Jujuy, en el año 1936, por funcionarios designados por el Gobierno de la Nación. Jujuy, Imprenta B. Buttazzoni.

»Publicación Oficial (1891). Memoria de la expedición a las regiones auríferas del Norte de la provincia de Jujuy. Expedida por la comisión nombrada por el superior gobierno de la Nación en abril de 1891. Buenos Aires, Imprenta y Librería de Mayo.

»Publicación Oficial (1917). Ley Número 207. Sobre patentes, registro de minas y decreto reglamentario. Jujuy, Talleres Gráficos del Estado

»Publicación oficial ordenada por la Subsecretaría de Hacienda (1923). Disposiciones atingentes con las concesiones mineras: Ley $N^{\circ} 368$, fundamentos de su origen y decretos del $9 y$ 25 de enero de 1923. Jujuy, B. Buttazzoni.

» Ramos, J. P. y A. Bonorino (1938). Querella por calumnias e injurias promovida por el ex gobernador de la provincia de Jujuy Dr. Arturo Pérez Alisedo contra el senador nacional Sr. Benjamín Villafañe. Buenos Aires, Talleres gráficos Marly.

» Riccardi, A. C. (2017). El desarrollo histórico de las exploraciones petroleras en la Jujuy. Anales de la Academia Nacional de Ciencias de Buenos Aires XLIX: 169-203.

» Rosas, L. V. y J. C. Ávila (2013). Desarrollo minero de Pirquitas, provincia de Jujuy. Ser. correl. geol., San Miguel de Tucumán 2: 33-42. Disponible en Internet: http://www.scielo. org.ar/scielo.php?script=sci_arttext\&pid=S166694792013000200004\&lng=es\&nrm=is o>. Consultado el: 15 de noviembre de 2017.

»Salizzi, E. (2014). “Gran minería” y transformaciones regionales en la Puna jujeña: el caso de Mina de El Aguilar (1936-1990). Estudios Sociales del Noa, 13: 47-66.

"Saravia, T. (1960). Geografía de la Provincia de Jujuy. Buenos Aires, Instituto Geográfico Militar

» Sica, G. y M. Ulloa (2006). “Jujuy en la Colonia. De la Fundación de la ciudad a la crisis del orden colonial” en Teruel, A. y M. Lagos (dirs), Jujuy en la historia. De la colonia al siglo $X X:$ 43-84. Jujuy, UNIHR/ EDIUNJu.

"Villafañe, B. (1926). El atraso del interior. Documentos oficiales del Gobernador de Jujuy pidiendo amparo para las industrias del Norte. Jujuy, Tip. Lib. B. Buttazzoni.

»Villafañe, B. (1938). El asesinato de Rafael Tauler perpetrado por las autoridades y amparado por la justicia. Un drama del far-west en la puna de Jujuy. La situación de Jujuy una vergüenza nacional. Buenos Aires, Imprenta Mercatali.

»Villafañe, B. (1940). El senador Villafañe ante la justicia del crimen y la conciencia nacional. S/D.

» Yrigoyen, M. R. (2007). Reseña sobre los conocimientos y la explotación de los hidrocarburos en Argentina antes de 1907. (Reedición de las notas publicadas en los números de Petrotecnia de marzo y abril de 1983). Petrotecnia, Revista del Instituto Argentino del Patróleo y del Gas (Febrero): 16-36. 